\title{
Role of Surface-State Nesting in the Incommensurate Reconstruction of Mo(001)
}

\author{
X. W. Wang, C. T. Chan, and K. M. Ho \\ Ames Laboratory-U.S. Department of Energy, Department of Physics, and Microelectronics Research Center, \\ Iowa State University, Ames, Iowa 50011
}

and

W. Weber

Institut für Nukleare Festkörperphysik, Kernforschungszentrum Karlsruhe, D-7500 Karlsruhe, Federal Republic of Germany

(Received 16 October 1987)

\begin{abstract}
First-principles total-energy calculations have been performed for the Mo(001) surface. Subsequent tight-binding calculations of the surface-phonon dispersion curves for the unreconstructed surface show that Fermi-surface nesting of surface states leads to a softening of surface modes near $\bar{M}$, with the maximum instability very close to the wave vector of the observed incommensurate reconstruction. Our results disagree strongly with the prevailing short-ranged force models for the incommensurate reconstruction on Mo(001).
\end{abstract}

PACS numbers: $68.35 . \mathrm{Ja}, 68.35$. Bs, 73.20.At

At low temperatures, the clean (001) surfaces of the isoelectronic transition metals $\mathrm{W}$ and Mo exhibit different reconstruction patterns: $\mathrm{W}(001)$ is commensurate $c(2 \times 2)$, yet $\mathrm{Mo}(001)$ has an incommensurate $c(2.2$ $\times 2.2)$ structure. ${ }^{1,2}$ The commensurate $\mathrm{W}(001)$ reconstruction has been studied in great detail. ${ }^{3,4}$ The purpose of the present paper is to study the even more challenging problem of the incommensurate $\mathrm{Mo}(001)$ reconstruction. While early theories have attributed both reconstructions to phonon instabilities driven by the nesting of surface electronic bands near the Fermi energy $E_{\mathrm{F}},{ }^{5}$ recent work emphasized the role of local bonding. ${ }^{6-11}$

Prevailing models ${ }^{12,13}$ for the incommensurate Mo(001) reconstruction invoke a simultaneous softening of the surface phonons at the $\bar{M}$ point of the surface Brillouin zone with lateral $\left(\bar{M}_{5}\right)$ as well as vertical $\left(\bar{M}_{1}\right)$ atomic displacements. Through a series of firstprinciples calculations, we show in this paper that the assumption of a soft $\bar{M}_{1}$ mode in these models ${ }^{12,13}$ is incorrect. Furthermore, using a tight-binding lattice dynamics technique, ${ }^{14}$ in combination with first-principles calculations, we will demonstrate that Fermi-surface nesting does play an important role in the production of the Mo(001) incommensurate reconstruction. However, the simple nesting picture is strongly modified because of electron-phonon matrix element effects.

The first-principles calculations are performed fully self-consistently within the local density-functional formalism with the Hedin-Lundqvist form ${ }^{15}$ of exchange correlation. Norm-conserving pseudopotentials ${ }^{16}$ are used and the Bloch wave functions are expanded in a mixed-basis set $^{17}$ comprising both plane waves and Gaussian orbitals localized on the atomic sites. This approach has been applied successfully to electronic, struc- tural, and lattice-dynamical properties ${ }^{18}$ of Mo and a large variety of transition-metal surfaces. The Mo(100) surface is modeled by a seven-layer slab. Fifteen $\mathbf{k}$ points in the irreducible surface Brillouin zone (SBZ) are used. ${ }^{19}$ The surface energy per surface atom and the work function for the ideal $p(1 \times 1) \mathrm{Mo}(100)$ surface are found to be 2.18 and $4.67 \mathrm{eV}$, respectively. A nine-layer slab gives $2.18 \mathrm{eV}$ for surface energy and $4.60 \mathrm{eV}$ for the work function. The small changes in these quantities indicate that the seven-layer slabs are adequate for computational purposes. The work function we obtained agrees well with the experimentally determined value of 4.53 $\mathrm{eV} .{ }^{20}$ The seven-layer $p(1 \times 1)$ slab is fully relaxed with use of Hellmann-Feynman forces, and the equilibrium coordinates are shown in Table I. The first-layer contraction of $10.7 \%$ is in very good agreement with those observed in LEED experiments $\left[(9.5 \pm 2) \%,{ }^{21} 11.5 \%{ }^{22}\right]$.

We have studied carefully the stability of the $p(1 \times 1)$ surface with respect to $\bar{M}_{1}$ (vertical) and $\bar{M}_{5}$ (lateral) surface-phonon distortions. In these studies, a $c(2 \times 2)$ unit cell is employed. We found that $\mathrm{Mo}(100)$ is quite stable with respect to a $\bar{M}_{1}$ surface phonon (surface atoms alternately moving up and down). By the frozen-

TABLE I. Relaxation and reconstruction for the seven-layer Mo $p(1 \times 1)$ and $c(2 \times 2)$ surface. $\Delta d_{12}$ is the percentage change of the interlayer distance between the first (top) and second layer. $\delta_{n}$ is the magnitude of reconstruction in the $\langle 11\rangle$ direction of the $n$th layer, given in percentage change of the lattice parameter $(4.44 \AA)$ of the $c(2 \times 2)$ unit cell.

\begin{tabular}{lrrrrrrr}
\hline \hline & $\Delta d_{12}$ & $\Delta d_{23}$ & $\Delta d_{34}$ & $\delta_{1}$ & $\delta_{2}$ & $\delta_{3}$ & $\delta_{4}$ \\
\hline$p(1 \times 1)$ & -10.7 & +2.7 & +0.3 & & & & \\
$c(2 \times 2)$ & -7.9 & +1.2 & +0.3 & 4.8 & 0.4 & -0.1 & $\sim 0$ \\
\hline \hline
\end{tabular}


phonon method, ${ }^{18}$ the effective surface force constant for the $\bar{M}_{1}$ mode is found to be $143 \mathrm{~N} / \mathrm{m}$ for the ideal (unrelaxed) surface, and $200 \mathrm{~N} / \mathrm{m}$ for the fully relaxed surface which as a smaller first-interlayer distance. We have repeated the calculation with a nine-layer slab as another test of slab thickness, and found that the effective surface force constant of the $\bar{M}_{1}$ mode for a seven-layer slab and a nine-layer slab differs by less than 3\%. Similar calculations on W(001) (Ref. 3) also predicted a stable $\bar{M}_{1}$ phonon. On the other hand, we found that (100) surface is unstable with respect to an $\bar{M}_{5}$ surface phonon with atomic displacements along the $\langle 11\rangle$ direction (Debe-King model), consistent with experimental findings that the reconstruction involves atomic displacements parallel to the surface. We have also found the equilibrium atomic positions within the Debe-King model [with a $c(2 \times 2)$ unit cell] by fully relaxing the system with Hellmann-Feynman forces. The multilayer relaxations and the displacement magnitudes along the 〈11) direction are shown in Table I. The first-layer contraction for the reconstructed surface is reduced with respect to the $p(1 \times 1)$ surface but is still quite substantial. Both relaxation and reconstruction magnitudes decay very rapidly into the inner layers. The fully relaxed $p(1 \times 1)$ surface is more stable than the ideal surface by $\sim 8 \mathrm{mRy} / \mathrm{surface}$ atom, while allowing for both relaxation and a $c(2 \times 2)$ "Debe-King" reconstruction lowers the energy further by $\sim 1 \mathrm{mRy} /$ surface atom. The $c(2 \times 2)$ reconstructed Mo(100) surface will gain more energy by going incommensurate so that our result is a lower bound for the reconstruction energy.

The calculations for the surface-phonon dispersion curves have been carried out for a seven-layer slab with use of a nonorthogonal tight-binding (NTB) parametrization of the electronic structure and electron-phonon matrix elements. ${ }^{23,24}$ Here, the phonon-dynamical matrix of the Mo slab, $D(q)$, is divided into $D_{b}$, a contribution of short-range nature representing the "bare" phonon frequencies, and $D_{2}$, the renormalization due to the conduction electrons. Using standard perturbation theory, we have

$$
D_{2}^{i \alpha, j \beta}(q)=-\sum_{\substack{\mathbf{k} \mu \mu^{\prime} \\ \mathbf{k}^{\prime}=\mathbf{k}+\mathbf{q}}} \frac{f_{\mathbf{k}^{\prime} \mu^{\prime}}-f_{\mathbf{k} \mu}}{\epsilon_{\mathbf{k} \mu}-\epsilon_{\mathbf{k}^{\prime} \mu^{\prime}}} g_{\mathbf{k} \mu, \mathbf{k}^{\prime} \mu^{\prime}}^{i \alpha} g_{\mathbf{k}^{\prime} \mu^{\prime}, \mathbf{k} \mu}^{j \beta},
$$

where $q$ denotes a surface wave vector, $g$ denotes the electron-phonon matrix elements coupling the electronic states identified by $\mathbf{k}$-point and band indices $\mathbf{k} \mu$ and $\mathbf{k}^{\prime} \mu^{\prime}$, and $f_{\mathbf{k} \mu}$ is the occupation number of an electronic state with eigenvalue $\epsilon_{\mathbf{k} \mu}$. The matrix elements $g^{i \alpha}$ contain the first-order gradients of the NTB integrals as well as the eigenvectors of the states $(\mathbf{k}, \mu),\left(\mathbf{k}^{\prime}, \mu^{\prime}\right)$. When we put $g=1$, the resulting term is the bare susceptibility $\chi_{0}(g)$ with a negative sign.

The NTB calculations require both realistic tightbinding parameters and the representation of the short- range part $D_{b}$ in terms of a Born-von-Kármán forceconstant model. We first repeated the bulk calculation as was done by Varma and Weber. ${ }^{23}$ The parameters for bcc Mo were obtained by a least-squares fit to the first-principles band structure. A nine-orbital $(s, p, d)$ basis was used, and the interactions were limited to first ( $1 \mathrm{nn}$ ) and second ( $2 \mathrm{nn})$ nearest neighbors. The bulk parameters were then used as the starting values for the slab calculation. The surface NTB parameters were determined by a fit to our first-principles band structure for a seven-layer slab with unrelaxed Mo(001) geometry. In the fit, only integrals involving first- and second-layer orbitals were allowed to change from their bulk values. We note that the dispersion of the first-principles $\bar{\Sigma}_{2}$ surface band is reproduced faithfully by the tight-binding fit; in particular, we obtained the same value of $k_{\mathrm{F}} \approx 0.62 \bar{M}$. The NTB gradients were obtained from Ref. 24. For the $D_{2}$ calculations a mesh of $78 \mathbf{k}$ points in the SBZ were used. The force constants for the shortranged $D_{b}$ were determined from calculations of bulk Mo phonons, utilizing the phonon data of Powell, Martel, and Woods. ${ }^{25}$ For simplicity, $D_{b}$ was not adjusted at the surface.

The phonon dispersion curves for unrelaxed Mo(001) are shown in Fig. 1. There appear three branches of surface phonons, two with $\bar{\Sigma}_{1}$ (sagittal-plane polarization) and one with $\bar{\Sigma}_{2}$ symmetry (shear-horizontal displace-

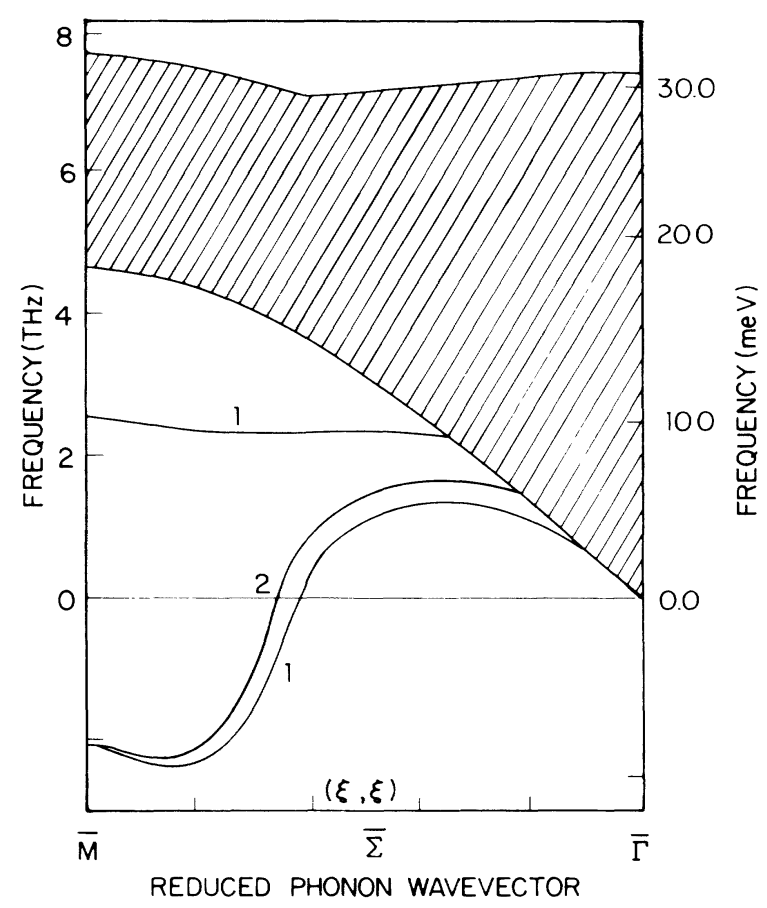

FIG. 1. Surface-phonon dispersion curves along $(\xi, \xi) \pi / a$ for $p(1 \times 1)$ Mo(001). The bulk-phonon continuum is indicated by the hatched area. The surface-phonon modes are the solid lines, with the $\Sigma_{1}$ modes being sagittal-plane (SP) polarized and $\Sigma_{2}$ mode being shear-horizontal $(\mathrm{SH})$ polarized. 
ments). The low-frequency $\bar{\Sigma}_{1}$ branch and the $\bar{\Sigma}_{2}$ branches exhibit mainly in-plane polarization; they have almost the same frequencies through most of the $\bar{\Gamma}-\bar{M}$ line and end at the doubly degenerate $\bar{M}_{5}$ phonon. These modes become unstable beyond $\xi \cong 0.5$, and the maximum instability is found near $\xi=0.83$. Phonon instabilities are also found on the $\bar{Y}$ line between $\bar{M}$ and $\bar{X}$ of the SBZ, but were less pronounced than those along $\bar{\Sigma}$.

The origin of the phonon instabilities near $\bar{M}$ is very similar to that for $\mathrm{W}(001){ }^{14}$ They arise from coupling to the surface electronic bands and resonances, mainly from the electronic $\bar{\Sigma}_{2}$ band. This surface band has Fermi vectors $k_{\mathrm{F}}=0.62 \bar{M}$ and $0.56 \bar{M}$ for $\mathrm{Mo}(001)$ and $\mathrm{W}(001)$, respectively. Its predominant component is the planar $d_{x^{2}-y^{2}}$ orbital. As a consequence, the $\bar{\Sigma}_{2}$ band has large electron-phonon matrix elements which, for symmetry reasons, are largest at $\bar{M}$ for atomic displacements with in-plane polarizations. However, the "bare" susceptibility of the slab $\chi_{0}(q)$ has its maximum near $\mathbf{G}-2 \mathbf{k}_{\mathrm{F}}$ ( $G$ being a surface reciprocal lattice vector). This corresponds to $\xi=0.76$ and 0.88 for Mo and W, respectively. The competition between matrix-element effects and $\chi_{0}(q)$ yields a maximum instability near $\xi=0.83$ for $\mathrm{Mo},{ }^{26}$ close to the observed wave vector of the $c(2.2 \times 2.2)$ reconstruction. For $\mathrm{W}$, the maximum instability occurs at $\xi=1.0$, and hence the reconstruction is commensurate. As a test, we found that by a lowering of $E_{\mathrm{F}}$ of the Mo(001) bands to the $k_{\mathrm{F}}$ value of W(001), the maximum instability is shifted to $\bar{M}$, indicating that the Fermi-surface nesting effect does play an important role in making the $\mathrm{Mo}(001)$ reconstruction incommensurate. The decrease of the $k_{\mathrm{F}}$ values from Mo to $\mathrm{W}$ is caused by a change in the overall electronic structure. Because of stronger relativistic effects in $\mathrm{W}$, the center of the (bulk) $s$ band is lowered relative to the $d$-band center, and thus the position of pure $d$ bands (such as $\left.\bar{\Sigma}_{2}\right)$ is higher, leading to the smaller $k_{\mathrm{F}}$ value.

The main difference between $\mathrm{Mo}(001)$ and $\mathrm{W}(001)$ is a dramatic reduction of the curvature in the dispersion of surface-phonon modes near $\bar{M}_{5}$. Analysis of the elements of the dynamical matrix reveals sharp variations as a function of wave vectors in the surface Brillouin zone, indicating that the interatomic forces responsible for the phonon instabilities extend to rather distant neighbors parallel to the surface. The diagonal term $D_{2}^{1 x, 1 x}(q)$, which describes the intralayer coupling of lateral displacements on the top surface layer, already has a dip near $\xi=0.83$ along $\bar{\Sigma}$. This shows that the incommensurate ground state occurs even in the absence of the coupling between the surface-phonon modes with inplane and out-of-plane displacements (the two $\bar{\Sigma}_{1}$ phonon branches near $\bar{M}_{5}$ ), in sharp contrast with current models ${ }^{12,13}$ in which that coupling is the key factor that drives the Mo reconstruction incommensurate. The small but repulsive interaction between the two $\bar{\Sigma}_{1}$ branches may, however, be the reason why the $\bar{\Sigma}_{1}$ (longitudinal wave) is pushed below the $\bar{\Sigma}_{2}$ (transverse wave) in the range of the unstable phonons. The possible coexistence of $\bar{\Sigma}_{1}$ and $\bar{\Sigma}_{2}$ types of distortion wave on a flat Mo(001) surface, as suggested by our results, would lead to a ground-state geometry consisting of almost square patches of $\bar{M}_{5}$ distortion, separated by solitonlike domain walls. This structure is consistent with the experimental LEED pattern. ${ }^{2}$ On a stepped surface, the narrow terrace may inhibit the formation of the $\bar{\Sigma}_{2}$ type of domain walls, leading to only $\bar{\Sigma}_{1}$ domain walls. ${ }^{27}$

In conclusion, we have presented a theoretical study of the Mo(001) $c(2.2 \times 2.2)$ surface reconstruction. This study represents a combination of first-principles totalenergy calculations and computations of the surfacephonon dispersion by a tight-binding method. Our results predict a stable $\bar{M}_{1}$ phonon and mainly planar surface-atom distortions, similar to the case of W(001), and the possible coexistence of $\bar{\Sigma}_{1}$ and $\bar{\Sigma}_{2}$ types of domain walls. This differs drastically from current models ${ }^{12,13}$ which predict large out-of-plane displacements of the surface atoms in the reconstructed surface, a consequence of an assumed soft $\bar{M}_{1}$ mode and a strong coupling along $\bar{\Sigma}$ between the two $\bar{\Sigma}_{1}$ branches. Further experiments are required to establish a correct picture of the incommensurate reconstruction on this surface.

We would like to thank Dr. E. Tosatti and Dr. C. Z. Wang for helpful discussions. Ames Laboratory is operated for the U.S. Department of Energy by Iowa State University under Contract No. W-7405-Eng-82. This work was supported by the Director for Energy Research, Office of Basic Energy Sciences, and the Microelectronics Research Center, Iowa State University, Ames, Iowa.

${ }^{1}$ M. K. Debe and D. A. King, Phys. Rev. Lett. 30, 708 (1977), and Surf. Sci. 81, 193 (1979).

${ }^{2}$ T. E. Felter, R. A. Barker, and P. J. Estrup, Phys. Rev. Lett. 38, 1138 (1977).

${ }^{3}$ C. L. Fu, A. J. Freeman, E. Wimmer, and M. Weinert, Phys. Rev. Lett. 54, 2261 (1985); C. L. Fu and A. J. Freeman, to be published.

${ }^{4}$ D. Singh, S. H. Wei, and H. Krakauer, Phys. Rev. Lett. 57, 3292 (1986).

${ }^{5}$ See, e.g., E. Tosatti, Solid State Commun. 25, 637 (1978); J. E. Inglesfield, J. Phys. C 11, L69 (1978); H. Krakauker, M. Posternak, and A. J. Freeman, Phys. Rev. Lett. 43, 1885 (1979).

${ }^{6}$ M. I. Holmes and T. Gustafsson, Phys. Rev. Lett. 47, 443 (1981).

${ }^{7}$ J. C. Campuzano, D. A. King, C. Somerton, and J. E. Inglesfield, J. Phys. C 14, 3099 (1981).

${ }^{8}$ J. E. Inglesfield, J. Phys. C 12, 149 (1979).

${ }^{9}$ K. Terakura, I. Terakura, and Y. Teraoka, Surf. Sci. 86, 535 (1979)

${ }^{10}$ M. Weinert, A. J. Freeman, and S. Ohnishi, Phys. Rev. 
Lett. 56, 2295 (1986).

${ }^{11}$ L. D. Roelofs and S. C. Ying, Surf. Sci. 147, 203 (1984).

${ }^{12}$ A. Fasolino, G. Santoro, and E. Tosatti, J. Phys. (Paris), Colloq. 42, C6-846 (1981); A. Fasolino and E. Tosatti, Phys. Rev. B 35, 4264 (1987); C. Z. Wang, E. Tosatti, and A. Fasolino, to be published.

${ }^{13} \mathrm{~V}$. Heine and J. J. A. Shaw, to be published.

${ }^{14}$ X. W. Wang and W. Weber, Phys. Rev. Lett. 58, 1452 (1987).

${ }^{15}$ L. Hedin and B. I. Lundqvist, J. Phys. C 4, 2064 (1971).

${ }^{16}$ D. R. Hamann, M. Schlüter, and C. Chiang, Phys. Rev. Lett. 43, 1494 (1979).

${ }^{17}$ S. G. Louie, K. M. Ho, and M. L. Cohen, Phys. Rev. B 18, 1774 (1979).

${ }^{18}$ C. L. Fu and K. M. Ho, Phys. Rev. B 28, 5480 (1983);

K. M. Ho, C. L. Fu, and B. N. Harmon, Phys. Rev. B 29, 1575 (1984).

${ }^{19}$ Thirty $\mathbf{k}$ points are used when we compare energy between $p(1 \times 1)$ and $c(2 \times 2)$ surfaces.

${ }^{20}$ S. Berge, P. O. Gartland, and B. J. Slagsvold, Surf. Sci. 43, 275 (1974).

${ }^{21}$ L. J. Clarke, Surf. Sci. 91, 131 (1980).

${ }^{22}$ A. Ignatiev, F. Jona, H. D. Shih, D. W. Jepsen, and P. M. Marcus, Phys. Rev. B 11, 4787 (1975).

${ }^{23}$ C. M. Varma and W. Weber, Phys. Rev. B 19, 6142 (1979).

${ }^{24} \mathrm{~W}$. Weber, in Electronic Structure of Complex Systems, edited by S. P. Phariseau and W. M. Temmerman, NATO Advanced Study Institue Series B, Vol. 113 (Plenum, Now V 1984), p. 345.

${ }^{25}$ B. M. Powell, P. Martel, and A. D. B. Woods, Can. J. I thys. 55, 1601 (1977).

${ }^{26} \mathrm{~A}$ nine-layer calculation yields a maximum instability at $\xi=0.9$.

${ }^{27}$ R. A. Barker, S. Semancik, and P. J. Estrup, Surf. Sci. 94, L162 (1980). 